\title{
The Design of An Efficient Traffic Scheduler with Fair Bandwidth-Sharing
}

\author{
for Wireless Multimedia Services*
}

\author{
Fu-Ming Tsou \\ Graduatc Institute of Commun. Engincering \\ National Taiwan University \\ d6942003@cc.ntu.cdu.tw \\ Zsehong Tsai \\ Graduate Institute of Commun. Enginecring \\ National Taiwan University \\ ztsai@cc.co.ntu.edu.tu
}

\begin{abstract}
In this paper, the Generalized Wireless Differentiated Fair Queneing (GWDFQ) algorithm is proposed to accommodate delay/jitter controls, and fair residual bandwidth sharing for real-time and non-real-time traffic streams simultaneously. The location-dependent channel error property, as appeared in most wireless networks, are considered in the algorithm and the temporary short error burst are compensated by the design of credits. The simulation results show GWDFQ can achieve excellent. performance, including timely delivery of real-time traffic, virtually loss-free transmission of non-real-time traffic, and fair usage of channel bandwidth among remote stations.
\end{abstract}

\section{INTRODUCTION}

$\mathrm{I}_{\mathrm{N}}^{\mathrm{N}}$ EVITABLY, the demands from customers in wireless access will lead to high volume of real-time (RT) and non-real-time (NRT) connectivity, which could often be beyond the available bandwidth. As a result, the issues of Quality of Service, fairness and pricing strategies should have expedited the emergence of service differentiation in such wireless access networks. However, due to the fact that characteristics of wireless channels can be very different from the wireline links, the traffic scheduler that satisfies the needs of wireless multimedia demands forms a complete new design.

A representative of the scheduling algorithms that specifically design for wireless access and to handle location-dependent error bursts is the idealized Wireless Fair-Queueing (IWFQ) algorithm proposed by Lu, Bharghavan and Srikant[1][2]. However, IWFQ does not consider the delay/jitter requirements in wireless multimedia applications. In addition, the guarantees for throughput and delay in IWFQ are tightly coupled, and may not satisfy the requirements of multimedia applications[3]. The Channel-condition Independent packet Fair Queueing (CIF-Q) algorithm[4] proposed by $\mathrm{Ng}$, Stoica and Zhang; the enhanced Class-Based-Queueing (enhanced CBQ) scheme[5] proposed by Fragouli et al., and the effort-limited fair (ELF) scheduling algorithm proposed by Eckhardt and Steenkiste[6] all provides long-term fairness and ensures delay and throughput guarantees for loss-free flows. However, either their implementation complexity is still too high for a cost-effective implementation or the flexible delay/jitler bounds may not be accommodated. Hence, when fairness, differentiated QOS in delay/jitter, and link utilization are all taken into consideration, it is necessary to redesign a new scheduling algorithm for wireless multimedia.

In this paper, we generalize the scheduling algorithm Wireless Differentiated Fair Queueing (WDFQ) proposed in [7] to accommodate flexible delay/jitter controls and fair residual bandwidth

\footnotetext{
- This work was in part supported by National Science Conncil of the R.O.C under Grant NSC89-2213-E-002-087, and by Ministry of Education of the R.O.C under Grant 89E-FA06-2-4-7.
}

sharing for RT and NRT traffic streams simultaneously. The GWDFQ not only possesses the advantages of WDFQ, such as 1) timely delivery of delay/jitter constrained RT traffic with controlled packet losses; 2) virtually error-free transmission of NRT traffic; 3) shared utilization of the residual bandwidth for both RT and NRT traffic streams, but also provides flexible delay/jitter controls for RT Traffic streams via incurring limited FIFO queues. In addition, the implementation of such traffic scheduler shall not require the use of sorter circuit and thus involve very limited complexity issues. The organization of this paper is as follows. In Section 2, the proposed traffic scheduler with fair residual bandwidth sharing is presented. Simulation results of RT and NR'T traffic under various scenarios are shown in Section 3. Our conclusions and future work are drawn in Section 4 .

\section{Generalized Wireless Differentiated Fatr QUEUEING DISCIPLINE}

\section{A. Minimum Bandwidth Guarantee for RT and NRT Traffic Streams}

The concept of minimum bandwidth guarantees and residual bandwidth sharing[8] is adopted in GWDFQ. In the following, we define some necessary notations. Due to the limitation of space, the detailed definitions of each notation and the workload calculation procedures are described in [7]. Note that underlying layer-2 PDU is assumed to be fixed and is called the air packet for simplicity in the following context. The time unit in GWDFQ is "slot" which is the time interval to transmit an air packet.

- $\left(B_{i}, M_{i}, \phi_{i}\right)$; the traffic profile of flow $i$ used in the service level agreement with respect to the air interface, consisting of the maximum burst size $B_{i}$, guaranteed minimum bandwidth $M_{i}$ and the share weighting factor of residual bandwidth $\phi_{i}$;

- $T$ : the length of a refreshing period, which is the period for service workload calculation;

- $t_{n}$ : the starting epoch of $n$-th refreshing period, and $t_{n}=$ $t_{n-1}+T$ for $n \geq 1$

- $W_{i}^{r}\left(t_{n}, t_{n+1}\right)$ : the reserved workload for a backlogged flow $i$ within the $n$-th refreshing period, which is the service workload satisfying the traffic profile of flow $i$;

- $W_{i}^{e}\left(t_{n}, t_{n+1}\right)$ : the extended workload for a backlogged flow $i$ within the $n$-th refreshing period, which is the workload contributed by the residual bandwidth observed within the $n$-th refreshing period;

- $W_{i}\left(t_{n}, t_{n+1}\right)$ : the total granted workload for a backlogged flow $i$ within the $n$-th refreshing period, which is the sum of $W_{i}^{r}\left(t_{n}, t_{n+1}\right)$ and $W_{i}^{e}\left(t_{n}, t_{n+1}\right)$ 
- $W_{i}^{E}\left(t_{n}, t_{n+1}\right)$ : the extra workload for a backlogged flow $i$, which is contributed by the total granted workloads of the flows under bad channel states at the starting epoch of the refreshing period;

- $\psi_{i}\left(t_{n}, t_{n+1}\right):$ the number of eligible air packets of flow $i$ within the $n$-th refreshing period.

For convenience, $W_{i}^{\top}\left(t_{n}, t_{n+1}\right), W_{i}^{\prime}\left(t_{r n}, t_{n+1}\right), W_{i}^{E}\left(t_{n}, t_{n+1}\right)$ and $W_{i}\left(t_{n}, t_{n+1}\right)$ are all normalized by the size of a single air packet.

When the $n$-th refreshing period starts and the integrity of an air packet is taken into considerations, the reserved workload of a backlogged flow $i$ can be calculated via the following recursive equation at $t_{\pi n}$ :

$$
\begin{gathered}
W_{i}^{r}\left(t_{n}, t_{n+1}\right)=\min \left\{\left\lfloor M_{i} T+B_{i}\right\rfloor,\left\lfloor(n+1) M_{i} T+B_{i}-\right.\right. \\
\left.\left.\sum_{j=0}^{n=1} \min \left\{W_{i}^{r}\left(t_{j}, t_{j+1}\right), \psi_{i}\left(t_{j}, t_{j+1}\right)\right\}\right\rfloor\right\}, n \geq 1 .
\end{gathered}
$$

It can be shown that eq. (1) is equivalent to the result of the leaky-bucket policing algorithm in [9]. However, the actual arrival rate may fluctuate, and the extended workload of a backlogged flow $i$ at $t_{n}$ is:

$$
\begin{aligned}
W_{i}^{e}\left(t_{n}, t_{n+1}\right) & =\left\lfloor\frac{\phi_{i}}{\sum_{j \in B\left(t_{n}\right)} \phi_{j}}(C T-\right. \\
& \left.\left.\sum_{j \in B\left(t_{n}\right)} \min \left\{W_{j}^{r}\left(t_{n}, t_{k+1}\right), \psi_{j}\left(t_{n}, t_{n+1}\right)\right\}\right)\right\rfloor .
\end{aligned}
$$

However, if flow $j$ is found to be with the bad channel state at $t_{n}$, the workload it granted, $W_{j}^{r}\left(t_{n}, t_{n+1}\right)$ and $W_{j}^{*}\left(t_{n}, t_{n+1}\right)$, should be distributed fairly to backlogged flows under good channel states, in order to achieve higher link utilization. Hence, backlogged flow $i$ with good channel state can obtain extra workload, $W_{i}^{E}\left(t_{n}, t_{n+1}\right)$, which is expressed as

$$
W_{i}^{E}\left(t_{n}, t_{n+1}\right)=\left\lfloor\frac{\phi_{i}}{\sum_{\substack{j \in B\left(t_{n}\right) \\ j \in G\left(t_{n}\right)}} \phi_{j}} \sum_{j \in E\left(t_{n}\right)}\left\{W_{j}^{r}\left(t_{n}, t_{n+1}\right)+W_{j}^{e}\left(t_{n}, t_{n+1}\right)\right\}\right]
$$

where $G\left(t_{n}\right)$ and $E\left(t_{n}\right)$ are the sets of flows under good channel state and under bad channel state at time $t_{n}$, respectively.

In addition, we use the concept of "credit" to compensate the loss or the overuse of bandwidth due to location-dependent errors and temporary short error burst. Detailed credit calculation algorithm and how it works can be referred to [7].

\section{B. Queneing Model of the GWDFQ Algorithm}

The queueing model in this paper is a generalized version of model in [7] and is shown in Fig. 1. Some important mechanisms in wireless networks, such as acknowledgement, channel state detection, etc., are assumed to be supported by the underlying MAC protocol. The jitter bounds ${ }^{1}$ of all flows in the Group RT-i are within $((i-1) T, i T]$ slot times, where $i=1, \cdots, N$. In GWDFQ, each flow is associated with a class of service with a set of pre-determined air-packet level QoS parameters, including delay/jitter, packet loss ratio, and residual bandwidth share, etc ${ }^{2}$.

In this model, non-real-time (NRT) traffic is assigned a special dedicated group, called Group NRT. The Head-of-Line (HOL) packet of a flow queue is called an eligible packet if it can be transmitted immediately without violating its delay bound

\footnotetext{
${ }^{1}$ In this paper, we follow the definition of jitter described in [10], where the jitter of a flow (or a connection) is defined by the maximum absolute difference in the delays experienced by any two packets on that flow.

${ }^{2}$ For NRT traffic, its delay and jitter limits are assigned infinite.
}

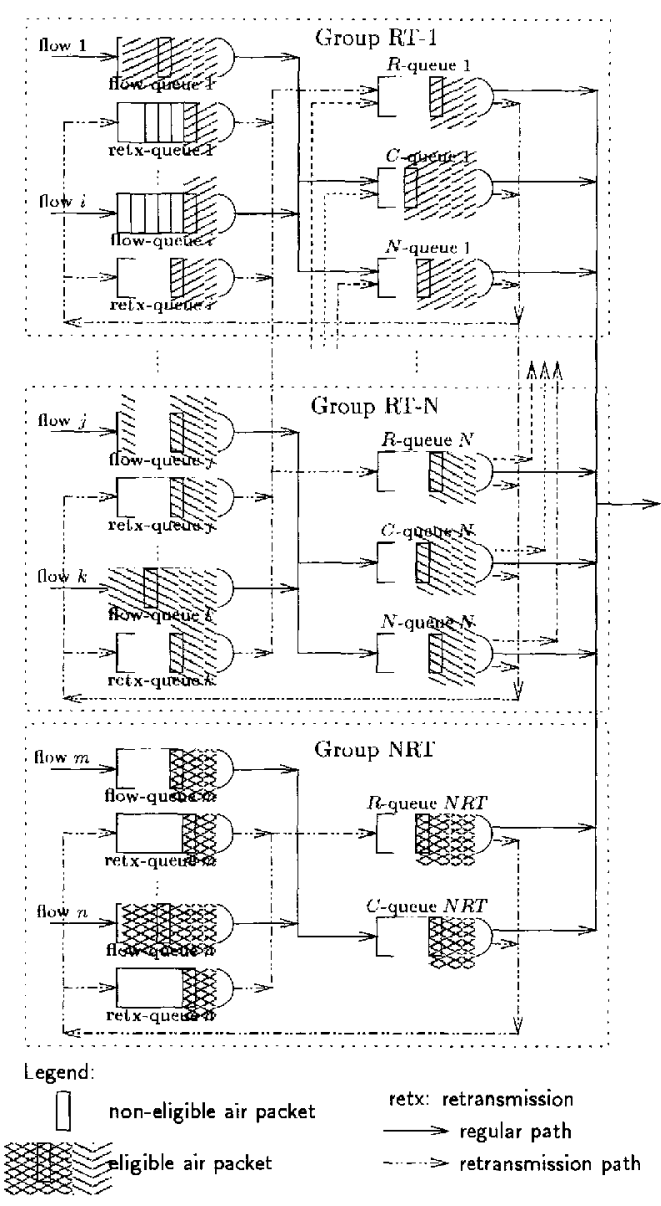

Fig. 1. The queueing model of the GWDFQ algorithm.

and packet jitter constraint. Each flow $i$ is assigned two dedicated FIFO queues, called flow-queue $i$ and retx-queue $i$. The function of flow-queve $;$ is to buffer the arriving air packets until they become eligible, while retx-queue $i$ buffers the eligible air packets whose channel states observed by the scheduler are under the "error" state now.

As far as RT traffic is concerned, three FIFO queues are required for each RT group at the output port, called $C$-queue, $N$-queue and $R$-queue, where $C, N$ and $R$ stand for conforming, nonconforming and retransmission, respectively. The $C$-queues of Group $i$, denoted as $Q_{C}^{(i)}$, buffers the air packets conforming to their service level agreements and the $N$-queue of Group $i$, denoted as $Q_{N}^{(i)}$, buffers the eligible but nonconforming air packets exceeding the service level agreements. In turn, the $R$-queue of Group $i$ (denoted as $Q_{R}^{(i)}$ ) buffers the eligible air packets whose flows encountered bad channel states previously and are ready to retransmit. The function and operation of $Q_{R}^{(i)}$ will be described in details later.It is noted that the delay bound and jitter bound of each RT flow hence has to be ceiled as an integer multiple of $T$.

On the other hand, for NRT traffic streams, only two extra FIFO queues, $Q_{C}^{(N F T)}$ and $Q_{F t}^{(N R T)}$, are needed. Nonconforming air packets of NRT traffic are still buffered in the corresponding flow queues. Hence, the $N$-queue is not necessary in Group NRT. This special mechanism for NRT traffic is to assure the 
air packet sequence integrity of each flow. Each time when the refreshing procedure starts, the air packets whose total traffic load is within the reserved workload and the extended worklond of each NRT flow are then moved to queue $Q_{C}^{(N R T)}$, before their transmission.

Due to lack of space, the mechanism of delay and jitter control for RT traffic streams is not described in this paper. However, it can be found in [7].

\section{Operations of the GWDFQ Algorithm}

Before describing the detailed operations of the GWDFQ algorithm, we briefly depict how GWDFQ deal with the retransmission mechanism under bad channel states.

Suppose that an air packet belonging to flow $i$ is picked from certain $C$-queue or $R$-queue to transmit when the current channel state is bad. Then, this air packet is moved back to retxqueue $i$ and the retransmission timer of flow $i$ with period $T_{i}^{r}$ is started. Before the retransmission timer expires, all eligible air packets belonging to flow $i$ are moved to retx-queue $i$. Therefore, it is not necessary for the scheduler to check the channel state slot by slot. Once the retransmission timer expires, the channel state of flow $i$ is updated and the eligible air packets in retx-queue $i$ are then moved to the corresponding $R$-queues. Air packets in $R$-queues are the cligible and conforming air packets that were not be served due to the bad channel states in previous refreshing periods. Hence, the $R$-queue is assigned highest service priority in a group to compensate their loss in bandwidth share. Last but not least, we have to note that the eligible air packets in $N$-queues are the air packets violating the traffic contracts. Thus, air packets in $N$-queues are of the lowest priority and may be subjected to packet discarding if conforming flow must be protected.

In the following, we describe the operations of GWDFQ. At each starting epoch of the refreshing period $t_{n}$, all eligible air packets in $Q_{R}^{(1)}, Q_{C}^{(1)}$ and $Q_{N}^{(1)}$ are discarded due to delay/jitter violations. Then, the eligible packets in $Q_{R}^{(i)}, Q_{C}^{(i)}$ and $Q_{N}^{(i)}$ are shifted to $Q_{R}^{(i-1)}, Q_{C}^{(i-1)}$ and $Q_{N}^{(i-1)}$, for $i=2, \cdots, N$. In turn, the reserved workload and the extended workload of each flow are calculated. Last, the eligible air packets from each flow queue are moved to the corresponding $C$-queues and $N$-queues according to their reserved workloads and the extended workloads. The eligible air packets conforming to traffic contracts are moved to $\mathrm{C}$-queues while nonconforming air packets are moved to $\mathrm{N}$-queues. For NRT traffic, conforming NRT packets are moved to $Q_{C}^{N R T}$ while nonconforming packets are buffered in the flow queues. Then, the eligible packets are serviced in the sequence of $Q_{R}^{(1)}, Q_{C}^{(1)}, \cdots, Q_{R}^{(N)}, Q_{C}^{(N)}, Q_{R}^{(N R T)}, Q_{C}^{(N R T)}, Q_{N}^{(1)}, \cdots$, $Q_{N}^{(N)}$. Via this service sequence, the delay/jitter requirements and the residual bandwidth shares can be accommodated simultaneously to RT multimedia streams.

\section{Simulation Results}

In this section, we evaluate the performance of the GWDFQ scheme for RT and NRT traffic streams. The examined performance metrics include the packet loss ratio, the bandwidth usage. Note that the packet loss ratio only accounts for those packets discarded due to delay or jitter violations. Because the buffer size is assumed infinite, no packet losses are due to buffer overflow.

Here, we assume the wireless channel follows IEEE 802.11[12] with link bandwidth is 10 Mbps at MAC layer. According to [12], we assume the overhead of MAC layer is 30 bytes in all simulation experiments. In addition, we assume the payload of the air packet is 128 bytes, which includes RTP header
12 bytes, UDP header 8 bytes, IP header 20 bytes and video frame data 88 bytes. Each video stream is a replay of "James Bond: Goldfinger" MPEG-1 video trace obtained from University Wuerzburg[13], with equally separated starting points within the 39996 frame positions. Since the frame rate is 24 frames/sec, each stream is equivalent, to a video of the length 1666.5 seconds. As for the statistical information of the video script trace are shown in Table I. The refreshing period is set to be $1.0 \mathrm{~ms}$ and all simulations lasts for $5 \times 10^{7}$ time slots, equivalent to 6320 seconds. We will show various of target delay constraints and jitter constraints can be supported easily. We have to note that as we mention the "bandwidth" or arrival/departure "rate," the protocol overheads from RTP layer to MAC layer are included.

\begin{tabular}{|c||c|c|c|}
\hline Frame Type & $\mathrm{T}$ & $\mathrm{P}$ & $\mathrm{B}$ \\
\hline \hline Mean frame length (air packets) & 118.82 & $\tilde{59.84}$ & $\mathbf{1 5 . 4 3}$ \\
\hline Variance of frame length & 1371.78 & $\mathbf{8 2 0 . 6 3}$ & $\mathbf{3 5 . 5 7}$ \\
\hline Max. frame length (air packets) & 348 & 296 & 79 \\
\hline \hline Overall mean ra.re & \multicolumn{3}{|c|}{$1.802 \mathrm{Mbps}$} \\
\hline
\end{tabular}

TABLE: 1

The general information of the MPEG video Tr.aCe in SIMUlations. ALL STATICS HAVE NVOLVED RTP, UDP AND IP LAYER OVERHEADS.

The error characteristic of the wireless channel is modeled by a 2-state Markov chain. If the channel state changes from GOOD state to BAD state suddenly during the air packet transmission period, the packet is received in error. The packet is received correctly otherwise. Every air packet received in error is assumed to be detected by the decoder.

In addition, we adopt the Priority FIFO algorithm as the baseline comparison, whose queueing model is shown in Fig. 2. The regulators, which serve as the front end packet processor, perform nothing except forwarding packets conforming the traffic profile $\left(B_{i}, M_{i}\right)$ to the high-priority output queue and forwarding nonconforming packets to the low-priority output queue, where $B_{i}$ is the maximum burst size and $M_{i}$ is the guaranteed minimum bandwidth.

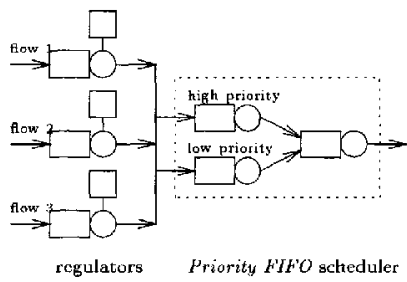

Fig. 2. The queueing model of the Priority FIFO algorithm.

\section{A. Experiment 1: Integrated Services with RT Traffic Streams} and NRT Traffic Streams

In this simulation scenario (see Fig. 3), we examine the transient behavior of the GWDFQ algorithm for NRT traffic streams. Two CBR flows (flow 1 and flow 2) are employed to model the RT traffic streams and their configurations are shown in Table II. Flow 3, serving as the background traffic, carries the NRT traffic stream which is a replay of LAN traffic trace obtained from the Lawrence Berkeley National Laboratory[14]. In total, the original trace is used to generate the traffic with 
average load equal to $7.2 \mathrm{Mbps}$. The output link bandwidth is also assumed $10 \mathrm{Mbps}$. We assume that the average time duration of the wireless channel at the GOOD (BAD) state is 10000 (1000) time slots. The retransmission periods of three flows are all 10 time slots and credit limits are 20 (air packets). In order to observe the behavior of bandwidth sharing more clearly: we set the average arrival rate of Flow 2 and Flow 3 much higher than their guaranteed minimum bandwidth $M_{i}$, and the packet loss ratios for two RT: flows are not considered in this simulation scenario.

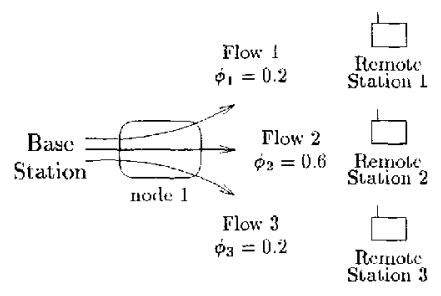

Fig. 3. Simulation model for Experiment 1

\begin{tabular}{|c|c|c|c|c|c|c|}
\hline & $\begin{array}{c}\text { Arrival } \\
\text { Rate } \\
(\mathrm{Mbps})\end{array}$ & $\begin{array}{c}\text { Reserved } \\
\text { BW Mi } \\
(\mathrm{Mbps})\end{array}$ & $\begin{array}{c}\text { MBs } \\
B_{i}\end{array}$ & $\begin{array}{c}\text { RBS } \\
\phi_{i}\end{array}$ & $\begin{array}{c}\text { Delay } \\
\text { Bound } \\
\text { (msec) }\end{array}$ & $\begin{array}{c}\text { Tit,ter } \\
\text { Bound } \\
(\mathrm{msec})\end{array}$ \\
\hline Flow 1 & 2.2 & 2.0 & 20 & 0.2 & 24 & 6 \\
\hline Flow 2 & 5.0 & 2.0 & 20 & 0.2 & 24 & 24 \\
\hline Flow 3 & 9.2 & 2.0 & 20 & 0.6 & N.A. & N.A. \\
\hline
\end{tabular}

TABT, II

Simulation CONFIgURATION FOR EXPERIMENT 1, WHERE N.A. STANDS FOR

"Not available." MBS stands for "MaXimum BuRst SizE" and its Unit' is air Packet. R.BS r.epresents "R.esidual Bandwidth Share."

If the channel states of three flows are all GOOD, according to the concept of GPS algorithm, it is easy to derive the the ideal granted-service rate of flow 2 and flow 3 should be $2.95 \mathrm{Mbps}$ and $4.85 \mathrm{Mbps}$. From Fig. 4 (a), we can observe that the channel states of three flows are all under GOOD states during the interval $[18.8,19.2] \mathrm{sec}$. And Fig. 4 (b) shows that three flows approaches their ideal granted-service rates under GWDFQ during the this interval. During the interval $[19.2,19.3]$ seconds, flow 1 enters BAD channel state. Hence, the ideal grantedservice rates of flow 2 and flow 3 during within this interval should be $3.5 \mathrm{Mbps}$ and $6.5 \mathrm{Mbps}$, respectively. Simulation results shown in Fig. 4 (b) verify that the granted-service rate of flow 1 is distributed fairly to flow 2 and flow 3 when flow 1 is under BAD channel state. After time 19.3 sec, the channel state of flow 1 becomes "GOOD" once again and it receive its granted-service rate right away. Other two flows also release their bandwidth granted from flow 1 when flow 1 was in $\mathrm{BAD}$ channel states. On the other hand, Fig. 4 (c) shows that although Priority FIFO can guarantee the minimum bandwidth, it cannot distribute the residual bandwidth to every backlogged flow fairly. Based on the simulation results obtained in Experiment 1, we conclude that GWDFQ not only provides QoS guarantees for RT traffic streams but also guarantees the bandwidth usage of NRT user groups following pre-determined traffic profiles.

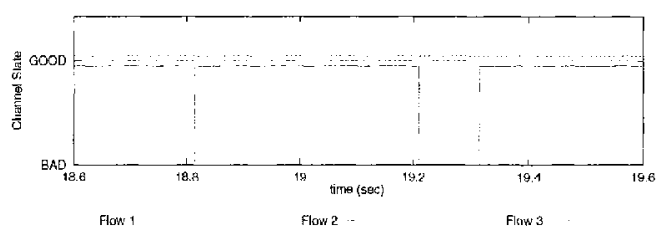

(a) Channel atate diagram of all flowa.

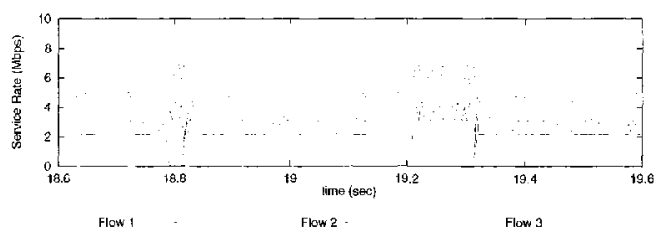

(b) Transient bandwidth sharing behaviors of GWDFQ with retransmission period 10 slots.

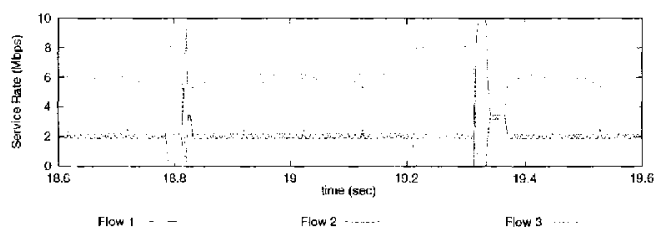

(c) Traneient bandwidth sharing behaviors of Priority FIFO, assuming perfect channel knowledge.

Fig. 4. Simulation results of Experimenr 1.

B. Experiment 2: The Influence of the Length of the Retransmission Period

In this section, we study the influence of the length of the retransmission period to find a best point to achicve the best performance via minimum processing overhead.

\begin{tabular}{|c|c|c|c|c|c|c|}
\hline & $\begin{array}{c}\text { Arrival } \\
\text { Rate } \\
(\mathrm{Mbps})\end{array}$ & $\begin{array}{c}\text { Reserved } \\
\text { BW } M_{i} \\
(\mathrm{Mbps})\end{array}$ & $\begin{array}{c}\text { MBS } \\
B_{i}\end{array}$ & $\begin{array}{c}\text { RBS } \\
\phi_{i}\end{array}$ & $\begin{array}{c}\text { Delay } \\
\text { Bound } \\
\text { (msec) }\end{array}$ & $\begin{array}{c}\text { Jitter } \\
\text { Bound } \\
(\mathrm{msec})\end{array}$ \\
\hline flow 1 & $\mathbf{3 . 3 4}$ & 2.0 & 10 & 0.6 & 24 & 24 \\
\hline flow 2 & 6.66 & 2.0 & 10 & 0.4 & N.A. & N.A. \\
\hline
\end{tabular}

TABLE III

Simulation CONfiguration for Experiment 2, where N.A. Represents "Not available" MBS stande for "Maximum Burst Stze" and its unit is air packet. RBS represents "Residual Bandwidth Share,"

The simulation model and configuration parameters are shown in Fig. 5 and Table III, respectively. The average lengths of "GOOD" period and "error" period are fixed as 1000 slots and 25 slots, respectively. The length of the retransmission period varies from 5 slots to 50 slots to investigate the influence of the length of the retransmission period. Flow 1 is a test video stream while flow 2 is an aggregated regular NRT flow driven by a LAN traffic trace.

Simulation results are shown in Fig. 6. We can observe that 


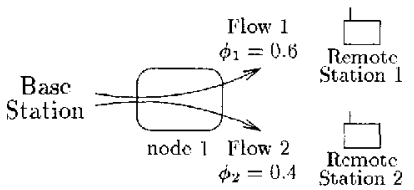

Fig. 5. Packet loss ratio of flow 1 under various lengths of retransmission periods.

if no control mechanism is adopted, the performance of the flow 1 is much worse under Priority FIFO than under GWDFQ in this comparison. Hence, in the following discussions, the performance of Priority FIFO are not included. On the other hand, in GWDFQ the packet loss ratio due to chamel errors increases slightly as the retransmission period is less than 25 slots, the average length of the error period. Therefore, we conclude that if we set the retransmission period too small compared to the average length of error period, the processing overhead will be high and the performance enhancement will not be sufficient. On the other hand, if the retransmission period is set too large, the performance degradation due to error period becomes significant. Hence, we recommend that the retransmission period should be set close to the observed average length of the error period as much as possible.

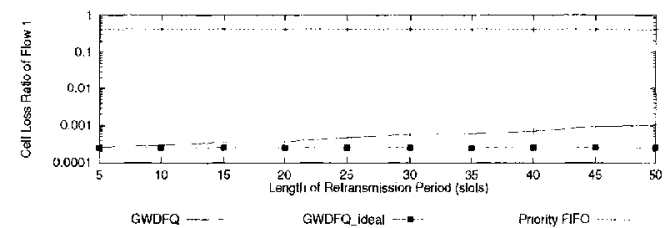

Fig. 6. The loss ratio of air packets in GWDFQ and Priority FIFO under various retransmission periods, where "GWDFQ_ideal" stands for the GWDFQ algorithm with full channel knowledge.

\section{Covalusions}

The proposed GWDFQ scheduling algorithm have been designed for transporting both RT streaming data and NRT traffic over wireless networks, and this algorithm can accommodate two different service levels (premium or regular) for $\mathrm{R} \Gamma$ or NRT traffic streams. As a result, the premium RT/NRT service and regular RT/NRT service can be accommodated simultaneously via the same scheduler architecture. We have also illustrated that the GWDFQ scheduler can provide fair access of residual bandwidth among all backlogged flows. As for supporting multiple service levels for RT traffic streams, we believe the GWDFQ scheme can be easily extended to accommodate this requirement without increasing too much implementation cost.

To summarize, timely delivery of RT traffic streams and virtually error-free transmission of NRT traffic are all well supported by GWDFQ. We believe that wireless multimedia services can be supported more easily by employing GWDFQenabled switches or base stations.

\section{REFERENCES}

[1] S. Lu, V. Bharghavan, and R. Srikant, "Fair Scheduling in Wireless Packet Networks," Proceedings of ACM SIGCOMM '97, pp. 63-74, 1997

[2] S. Lu, V. Bharghavan, and R. Srikant, "Fair Scheduling in Wireless Packet Networks," IEEE/ACM Trans. Networking, vol. 7 , no, 4, pp. 473-489, Aug. 1999 .
[3] I. Stoica, H. Zhang, and T. S. E. NE, "A Hierarchical Fair Service Curve Algorithrn for Link-Sharing, Real-Time and Priority Services," ACM Comput Commur., vol. 27, pp. 247-262, Oct. 1997.

[4] T. S. E. Ng. T. Stoica, and H. Thang, "Packet Fair Queveing Algorithms for Wireless Networks with Location-Dependent Errors," Proceedings of IEEE NFOCOM ' 98, Mar. 1998

[E] C. Fragouli, V. Sivaraman, and M. B. Srivastava, "Controlled Multimedia Wireless Link Sharing via Enhanced Class-Based Queueing with Channe?State-Dependent Packet Scheduling," Proceedings IEEE INFOCOM '98. Mar. 1998 .

[6] D. A. Fekhardt and P. Steenkist,e, "Effort-limited Fair (ELF) Scheduling for Wireless Networks," Proceedings of IEEE INFOCOM 2000, pp. 1097-1106, 2000.

[7] F.-M. Tson, H.-B. Chion and Z. Tsai, "WDFQ: An Efficient Traffic Scheduler with Fair Bandwidth-Sharing for Wireless Multimedia Services," to appear in IEICE Trans. Commun,, Apr. 2001.

[8] ATM Forum Technical Committee, "Traffic Management Specification Version 4.1," AF-TM-0121.000, Mar, 1999.

[9] ITU-T Recommendation 1.371, Traffic Control and Congestion Corltrol in B-ISDN, Aug. 1996.

[10] D. Verma, H. Zhang, and D. Ferrari, "Delay Jitter Control for Real-Time Communication in a Packet Switching Network," Proceedings of IEEE Tricomm 91, pp. 35-46, Aprl. 1991.

[11] J. Liebeherr and D, E. Wrege, "Priority Quene Schedulers with Approximate Sorting in Output Buffered Switches," IEEE J. Select. Areas Commun., vol. 17, no. 6, Pp. 1127-1144, June 1999.

[12] IEEE 802.11: IEEE Standard for Wireless LAN Medium Access Control (MAC) and Physical Layer (PHY) specifications, June 26, 1997.

[13] O. Rose, MPEG-1 Video Trace, James Bond: Goldfinger, Institute of Computer Science III, University Wuerzburg, $\mathrm{ft}_{\mathrm{p}} / / \mathrm{ft}$ p-info3.informatik.uniwuerzburg.de/pub/MPEG/traces, 1995.

[14] W. Leland and D. Wilson, ftp://ita.ee.lbl.gov/traces/BC-pAug89.TL.Z, 1989. 